\title{
$\Gamma$-Convergence Approximation to Piecewise Smooth Medical Image Segmentation
}

\author{
Jungha $\mathrm{An}^{1,2}$, Mikael Rousson ${ }^{2}$, and Chenyang $\mathrm{Xu}^{2}$ \\ ${ }^{1}$ Institute for Mathematics and its Applications (IMA), University of Minnesota, \\ Minneapolis, MN, USA \\ ${ }^{2}$ Imaging and Visualization Department, Siemens Corporate Research, Princeton, NJ, USA
}

\begin{abstract}
Despite many research efforts, accurate extraction of structures of interest still remains a difficult issue in many medical imaging applications. This is particularly the case for magnetic resonance (MR) images where image quality depends highly on the acquisition protocol. In this paper, we propose a variational region based algorithm that is able to deal with spatial perturbations of the image intensity directly. Image segmentation is obtained by using a $\Gamma$-Convergence approximation for a multi-scale piecewise smooth model. This model overcomes the limitations of global region models while avoiding the high sensitivity of local approaches. The proposed model is implemented efficiently using recursive Gaussian convolutions. Numerical experiments on 2-dimensional human liver MR images show that our model compares favorably to existing methods.
\end{abstract}

\section{Introduction}

Extracting structures of interest through image segmentation is an important task in medical imaging. Image segmentation is especially needed for better visualization, quantification of diseases, and planning an intervention. This task of segmenting a given region from the rest of the image usually relies on image information that can be edge-based, region-based, or a combination of both. MR imaging is a modality where these criteria are not sufficient. In MR imaging, various scanning parameters are used to highlight different living tissues. As a result, image characteristics can vary significantly from one acquisition to another, particularly due to inhomogeneities in the radio frequency field. If one wants to define a generic segmentation problem, robustness to these intensity variations, often referred to as bias field, is mandatory. One may want to correct for these artifacts prior to the segmentation [14], but both problems are intrinsically dependent on each other. In [15], an Expectation-Maximization technique was proposed to estimate the bias field jointly with the segmentation. In this paper, a more straightforward approach is proposed by considering a segmentation model that is naturally robust to smooth spatial variations of the intensity.

Most of recent geometric segmentation methods use region statistics to model intensity distributions of the objects and the background. These global models have shown to be more robust to initialization and noise than local or edge-based approaches, but the assumption of a global intensity distribution is not relevant in most cases, particularly with MR images like the one shown in Figure 1. Interestingly, these models are modifications of the seminal work of Mumford and Shah (MS) [9] that originally did not make such an assumption of regional distributions, but simply intended

N. Ayache, S. Ourselin, A. Maeder (Eds.): MICCAI 2007, Part II, LNCS 4792, pp. 495-502, 2007.

(C) Springer-Verlag Berlin Heidelberg 2007 


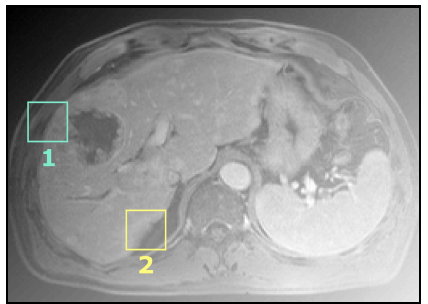

MR image

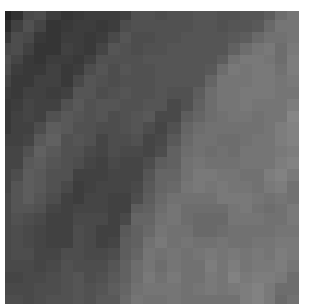

zoom 1

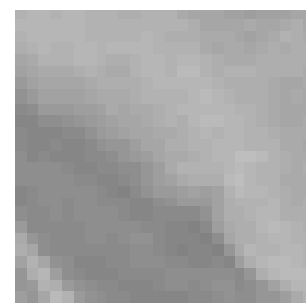

zoom 2 (rotated by $\left.180^{\circ}\right)$

Fig. 1. High bias in MR images - We zoom in two different parts of the image shown on the left. The second zoom is rotated by 180 degrees, so that we can see that the intensity level outside can be lower than the intensity inside. Our perceptual vision may tell us the contrary.

to recover a piecewise smooth approximation of the image. For this purpose, the image domain $\Omega$ is decomposed in a set of regions separated by a smooth boundary $\Gamma$. Then, minimizing the MS functional requires the joint estimation of the boundary $\Gamma$ and the "ideal" smooth image. However, such loose assumptions on the objects to recover make this approach very sensitive to the initialization of $\Gamma$ and computationally expensive.

There have been several approaches developed to approximate the MS model numerically. Chan and Vese first proposed a level set formulation [10] of the simplified piecewise constant MS model in [4], before extending it to the more general piecewise smooth model [5.7]. The first model has the advantage of being relatively robust and has a low computational complexity, but it is also too simplistic for most applications. The second one is an elegant numerical implementation of the general MS model, but still depends highly on the initial conditions and has a high computational complexity. As an alternative, Ambrosio and Tortorelli [1] approximate the measurement of the length term in the MS model by a quadratic integral of an edge signature function. In [5]7, the segmentation is represented by characteristic functions using phase field models [3 8 [12 13]. Finally, in [2 12], the piecewise constant MS model is reformulated using a $\Gamma$-Convergence approximation, which motivates our proposed model in this paper.

A new region based variational formulated model for a piecewise smooth image segmentation is proposed. Image segmentation is obtained using a $\Gamma$-Convergence approximation and multi-scale local statistics. The proposed model is motivated by [2 4|5 5|11|12]. Our model differs from commonly used ones, like [2 4|5|12] by relying on local intensity averages rather than global statistics. Our formulation is closely related to [611]. The improvements brought by our model are twofolds: we introduce a $\Gamma$-Convergence approximation and we integrate multiple scales for local intensity models. We also present a validation on 2-dimensional slices of human liver MR images. This paper is organized as follows: In Section 2, both the single and multi-scale models are proposed. The Euler-Lagrange equations of the suggested model are also presented in this section. Experimental results of the proposed model with comparison to existing models are shown in Section 3. Finally, in Section 4, conclusions are drawn and future work is stated. 


\section{Description of the Proposed Model}

In this section, we introduce a $\Gamma$-Convergence approximation motivated by [212] for the piecewise-smooth model proposed in [11]. For clarity, a single scale model is derived first and then the general model with multi-scale is presented.

\subsection{Single Scale Model}

Image Segmentation is obtained using a $\Gamma$-Convergence approximation and single scale local statistics. Two phases are assumed for the simplicity of our model. The model aims at finding the phase field $\theta$ by minimizing the following energy:

$$
\begin{aligned}
E(\theta)= & \lambda \int_{\Omega} f(\theta)\left(I-u_{\text {in }}(\theta)\right)^{2}+(1-f(\theta))\left(I-u_{\text {out }}(\theta)\right)^{2} d x \\
& +(1-\lambda) \int_{\Omega} \varepsilon_{1}|\nabla f(\theta)|^{2}+\frac{f(\theta)^{2}(1-f(\theta))^{2}}{\varepsilon_{1}} d x,
\end{aligned}
$$

where $I$ is a given image, $\Omega \in \mathbb{R}^{3}$ is its domain, $f$ is a smooth version of the Heaviside function, $\varepsilon_{1}$ is a positive parameter, and $0<\lambda<1$ is a parameter balancing the influence of the two terms in the model. Following [11], $u_{\text {in }}$ and $u_{\text {out }}$ are expressed as local weighted intensity averages that can be obtained by Gaussian convolutions:

$$
u_{\mathrm{in}}(\theta)=\frac{g_{\sigma} *[f(\theta) I]}{g_{\sigma} * f(\theta)} \text { and } u_{\mathrm{out}}(\theta)=\frac{g_{\sigma} *[(1-f(\theta)) I]}{g_{\sigma} *(1-f(\theta))},
$$

where $g_{\sigma}$ is a Gaussian kernel with standard deviation $\sigma$, and "*" stands for the convolution in the image domain $\Omega$.

This model is an efficient approximation of the general piecewise smooth MS model. One can point out that it becomes equivalent to the piecewise constant model [2]4], when the variance $\sigma$ goes to infinity. A piecewise smooth approximation of the image can accommodate a wider range of problems than its piecewise constant counterpart. In particular, it is well-suited for image modalities with bias, as it is often the case in MRI.

In the second term of Equation 11, $\varepsilon_{1} \ll 1$ controls the transition bandwidth. The $\Gamma$ Convergence is used to approximate the length term in the MS model. In the theory of $\Gamma$-Convergence [1], the length of $\Gamma$ is approximated by a quadratic integral of an edge signature function $p$. This model is combined with a double-well potential function $W(p)=p^{2}(1-p)^{2}$ with $p \in H^{1}(\Omega)$. As $\varepsilon_{1} \rightarrow 0$, the first term penalizes unnecessary interfaces and the second term forces the stable solution to take values of 1 or 0 . The second term in our model is followed from [2|12|13]. For details on phase field models and double-well potential functions, please refer to [2|12 13].

\subsection{Multi-scale Model}

The model considered so far is based on local intensity averages $\left(u_{\text {in }}, u_{\text {out }}\right)$. The locality of these terms is determined by the standard deviation $\sigma$ of the Gaussian kernel $g_{\sigma}$, which has been supposed to be the same for all pixels. This may be a limitation, since 

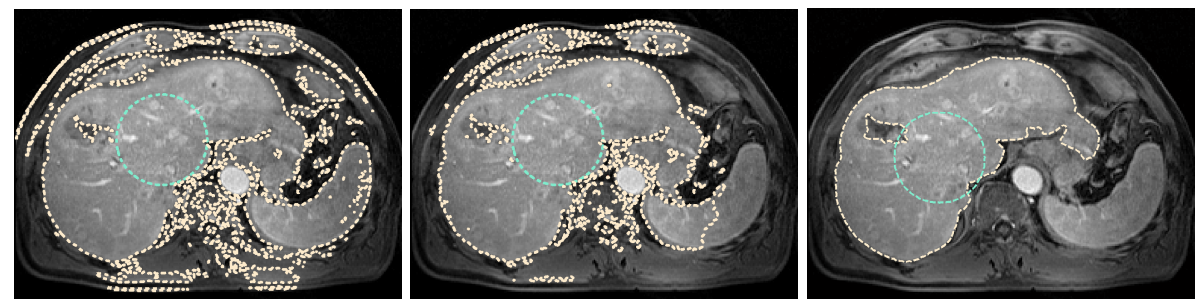

Fig. 2. Comparison between 3 models - From left to right : Chan and Vese model, An and Chen model, and our model with a single scale $(\sigma=15)$. In this figure, as well as in the next ones, the initialization is shown in dark (light green) and the final segmentation in bright (yellow).

$\sigma$ should be big enough to attract the contour to edges and small enough to detect weak edges. To resolve this problem, $\sigma$ should be defined pixel-wise. However, this would highly increase the complexity of the algorithm. An intermediate step is to consider only two different scales $\sigma_{1}$ and $\sigma_{2}$, and combine them at each pixel with different weights. Let $\sigma_{1}<\sigma_{2}$. We want to use the region term with $\sigma_{1}$ where the image gradient is high, and with $\sigma_{2}$ where it is low. This leads us to the following modification of the first term of Equation (1):

$$
\begin{aligned}
& E_{\text {region }}(\theta)=\lambda \int_{\Omega} f(\theta)\left[g(|\overline{\nabla I}|)\left(I-u_{\sigma_{1}, \text { in }}(\theta)\right)^{2}+(1-g(|\overline{\nabla I}|))\left(I-u_{\sigma_{2}, \text { in }}(\theta)\right)^{2}\right] d x \\
& +\lambda \int_{\Omega}(1-f(\theta))\left[g(|\overline{\nabla I}|)\left(I-u_{\sigma_{1}, \text { out }}(\theta)\right)^{2}+(1-g(|\overline{\nabla I}|))\left(I-u_{\sigma_{2}, \text { out }}(\theta)\right)^{2}\right] d x,
\end{aligned}
$$

where $g(|\overline{\nabla I}|)$ acts as an edge detector, $|\overline{\nabla I}|$ stands for the magnitude of the gradient of a smoothed version of the image that is normalized between 0 and 1 , and the function $g$ is an increasing function from $[0,1]$ to $[0,1]$. This permits us to assign the lower sigma to low gradient edges that enables the model to capture weak boundaries.

\subsection{Energy Minimization}

To obtain the best segmentation according to our piecewise smooth model, we need to minimize the corresponding energy with respect to the phase field $\theta$. For this purpose, we need to derive the Euler-Lagrange equations with respect to $\theta$. For clarity, we present the gradient descent obtained for the single scale energy (1):

$$
\begin{aligned}
\frac{\partial \theta}{\partial t}= & -\lambda f^{\prime}(\theta)\left[\left(I-u_{\text {in }}(\theta)\right)^{2}-q_{\text {in }}-\left(I-u_{\text {out }}(\theta)\right)^{2}+q_{\text {out }}\right] \\
& +(1-\lambda)\left[2 \varepsilon_{1} \operatorname{div}\left(\nabla \theta\left(f^{\prime}(\theta)\right)^{2}\right)-2|\nabla \theta|^{2} f^{\prime}(\theta) f^{\prime \prime}(\theta)\right] \\
& -(1-\lambda)\left[\frac{f(\theta)(1-f(\theta))(1-2 f(\theta)) f^{\prime}(\theta)}{\varepsilon_{1}}\right], \text { in } \Omega \\
\frac{\partial \theta}{\partial n}= & 0, \text { on } \partial \Omega,
\end{aligned}
$$




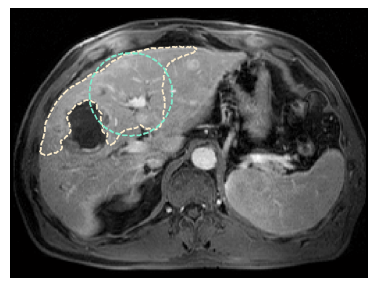

$\sigma=4$

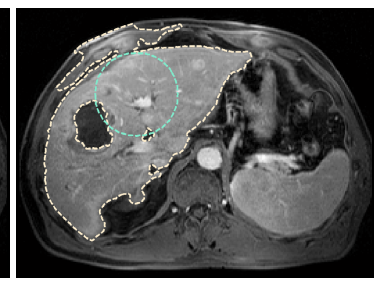

$\sigma=12$

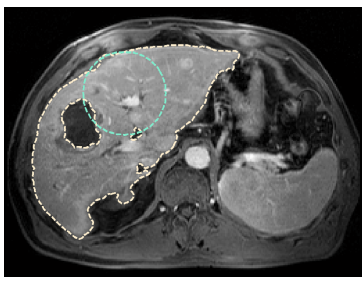

$\left(\sigma_{1}=4, \sigma_{2}=12\right)$

Fig. 3. Single scale versus multi-scale - When a single scale is considered for the computation of local averages, the contour either gets stuck (low $\sigma$ ) or leaks (high $\sigma$ ). Using the multi-scale formulation that combines a low $\sigma$ for high gradient areas with a high $\sigma$ for homogeneous regions, the contour does not get stuck in homogeneous regions and still correctly segments weakly defined edges.

where the terms $q_{\text {in }}$ and $q_{\text {out }}$ have the expressions:

$$
\left\{\begin{array}{l}
q_{\text {in }}=I\left[g_{\sigma} * \frac{2 f(\theta)\left(I-u_{\text {in }}(\theta)\right)}{g_{\sigma} * f(\theta)}\right]-g_{\sigma} * \frac{\left.2 f(\theta)\left(I-u_{\text {in }} \theta\right)\right) u_{\text {in }}(\theta)}{g_{\sigma} * f(\theta)} \\
q_{\text {out }}=I\left[g_{\sigma} * \frac{2(1-f(\theta))\left(I-u_{\text {out }}(\theta)\right)}{g_{\sigma} *(1-f(\theta))}\right]-g_{\sigma} * \frac{\left.2(1-f(\theta))\left(I-u_{\text {out }} \theta\right)\right) u_{\text {out }}(\theta)}{g_{\sigma} *(1-f(\theta))} .
\end{array}\right.
$$

The gradient descent of the multi-scale model can be easily obtained by analogy.

\section{Numerical Results}

In this part, we show different numerical results on 2-dimensional human liver MR images. Each experiment consists of contouring the liver boundary in axial slices. Several images include tumors inside the liver, which will be considered as background.

These experiments include the minimization of the single scale and multi-scale criterion. They are minimized by finding a steady-state solution of the corresponding evolution equations. A finite difference scheme is applied for discretization. $f(\theta)=$ $\frac{1}{2}\left\{1+\frac{2}{\pi} \arctan \left(\frac{\theta}{\varepsilon}\right)\right\}$ is used in the numerical calculation and $g$ was empirically chosen as $g(v)=\alpha v$ for $0 \leq v<\frac{1}{\alpha}$, and 0 otherwise. Parameters $\left(\lambda, \varepsilon, \varepsilon_{1}, \alpha\right)$ are set to $(0.5,0.01,0.01,0.3)$ empirically for all the experiments.

\subsection{Comparisons and Experiments}

In Figure 2, we compare the proposed model with existing methods [2]4]. The piecewise constant models use global image information and cannot discriminate the average intensity of the liver with neighboring structures. This is mainly due to the black region around the body that corrupts the mean value of $u_{\text {out }}$. Using local averages makes the approach robust to any disturbing factors far from the object of interest.

In the second experiment, shown in Figure 3, we show the influence of the parameter $\sigma$ in the single scale version of our model. With too low values of $\sigma$, the contour gets stuck in homogeneous regions, because local intensity averages inside and outside the 

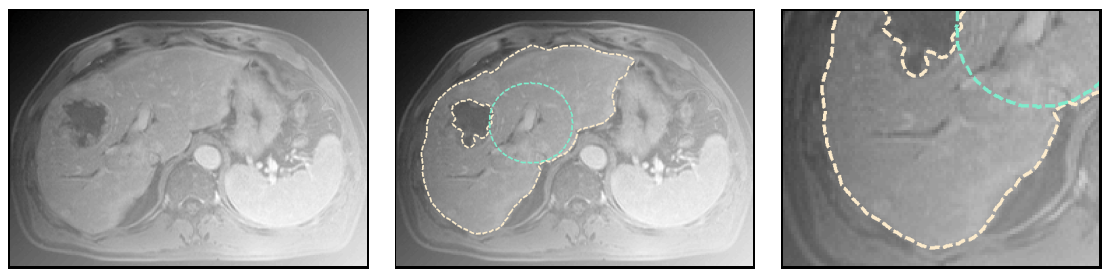

Fig. 4. Result on an MR image with high synthetic bias - From left to right: modified image, final segmentation, and a zoom on this segmentation $\left(\sigma_{1}=4\right.$ and $\left.\sigma_{2}=8\right)$. Our multi-scale approach is able to deal quite easily with this artifact. This is an important advantage since this type of intensity variation is very common in MR imaging.
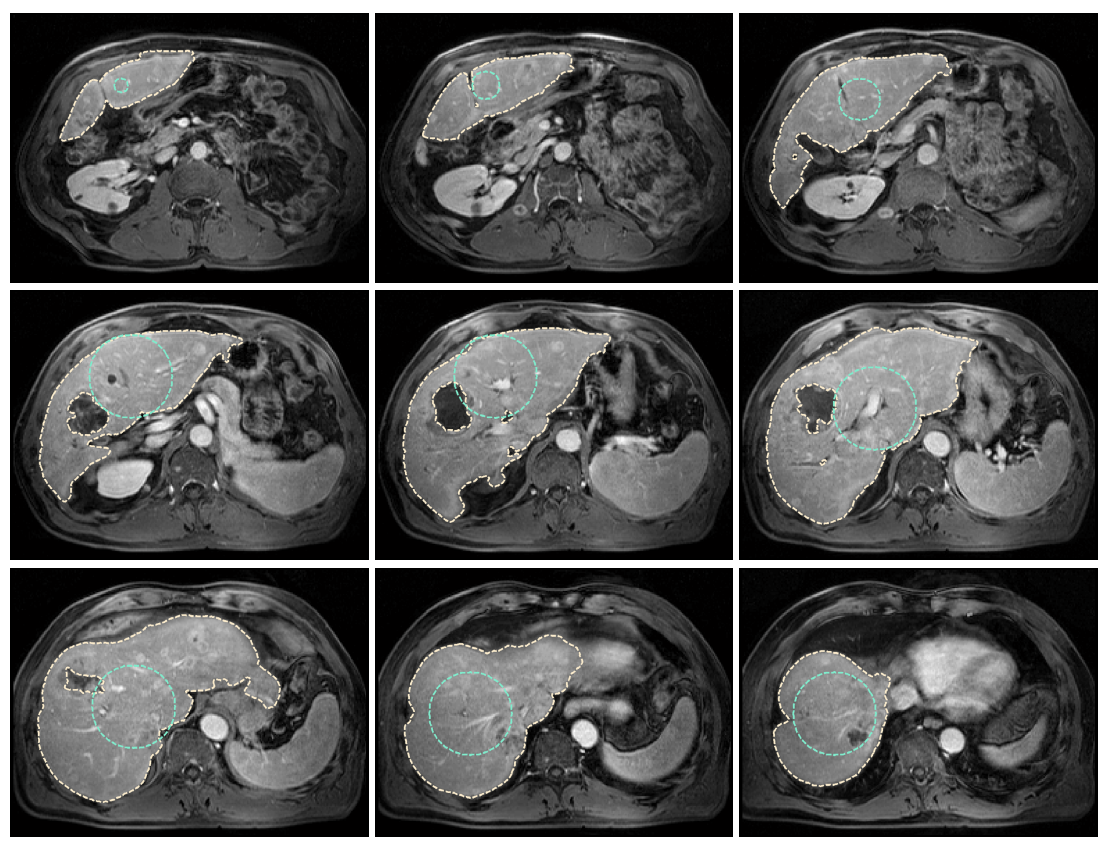

Fig. 5. Liver segmentation in an MR volume - Segmentations obtained on 2-dimensional slices of an MR volume ( $\sigma_{1}=4$ and $\left.\sigma_{2}=8\right)$

contour are almost identical. On the other side, if $\sigma$ is too big, the contour does not stop in homogeneous regions, but it is not able to capture weakly defined boundaries. The reason is that structures outside the liver will bias the outside local mean, outweighing the intensity gradient of weak edges. The last image of this figure shows the result obtained with the multi-scale formulation. In this case, we need to choose two smoothing parameters: $\sigma_{1}$ and $\sigma_{2}$. Rather than complicating the approach, this allows us to specify one locality for pixels close to edges, another one for homogeneous regions, both being combined together to avoid any sharp decision (see Equation (2)). Figure 4 shows the numerical result on MR image with high synthetic bias. 

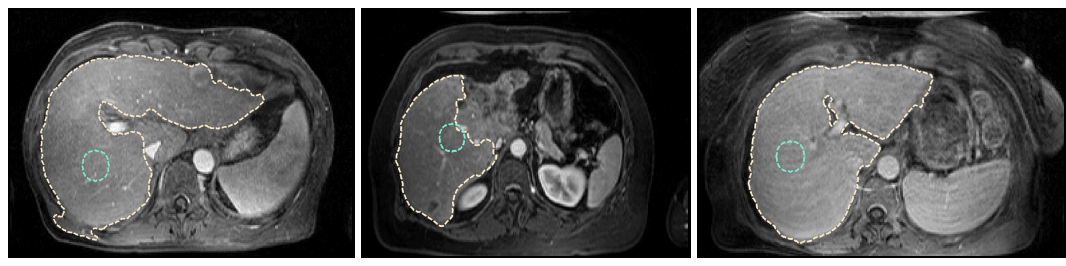

Fig. 6. Liver segmentation in an MR volume - Segmentations obtained on 2-dimensional slices of 3 other MR volumes ( $\sigma_{1}=4$ and $\sigma_{2}=8$ )

\subsection{Validation}

The next experiment is a validation on a complete MR volume. The algorithm has been tested on each slice intersecting the liver. Figure 5 shows the results obtained on a few slices of one volume, while Figure 6 shows 3 slices segmented in 3 other patients. Most of the segmentations follow the liver boundary, even if there is a bias and weakly defined edges in the image. This approach is still not perfect since a few small leakages are visible. To validate more quantitatively the results, we compared them to the segmentations given by an expert. For each slice, we estimate the Dice coefficient and the average surface distance. As shown in Table 3.2, the algorithm shows very promising results.

\begin{tabular}{|c|c|c|}
\hline Image \# & Dice coefficient \pm stdv & Average contour distance \\
\hline \hline 1 & $0.89 \pm 0.05$ & $2.24 \mathrm{~mm}$ \\
2 & $0.86 \pm 0.06$ & $2.75 \mathrm{~mm}$ \\
3 & $0.87 \pm 0.05$ & $3.06 \mathrm{~mm}$ \\
4 & $0.92 \pm 0.02$ & $2.00 \mathrm{~mm}$ \\
\hline \hline Average & $0.89 \pm 0.04$ & $2.51 \mathrm{~mm}$ \\
\hline
\end{tabular}

Fig. 7. Validation of our model on 2-dimensional slices of $4 \mathrm{MR}$ images - The same parameters were used for each image. The voxel size in each slice $1.4 \mathrm{~mm} \times 1.4 \mathrm{~mm}$ and the inter-slice distance is $3 \mathrm{~mm}$.

Regarding the speed of the algorithm, 1000 iterations were run until the convergence reached in around 20 seconds on a standard computer on a $256 \times 200$ image. Depending on the parameters, 1000 iterations is usually enough to get to a steady-state.

\section{Conclusions and Future Work}

Multi-scale local intensity statistics have been introduced in a variational formulation for the segmentation of liver MR images. Our approach combines $\Gamma$-Convergence approximation with a new multi-scale piecewise model. We have shown that it was able to deal directly with MR images including spatial intensity inhomogeneities. Numerical results show the effectiveness of the proposed model. Future works will include a validation on $3 \mathrm{D}$ data and learning the different parameters that are now chosen empirically. 


\section{Acknowledgments}

We thank Bernhard Geiger for providing liver MR images, as well as Ali Khamene and Frank Sauer for their support. This work is funded by Siemens Corporate Research and Institute for Mathematics and its Applications (IMA) at the University of Minnesota.

\section{References}

1. Ambrosio, L., Tortorelli, V.: Approximation of functionals depending on jumps by elliptic functionals via $\Gamma$-convergence. Communications on Pure and Applied Mathematics 43, 999 1036 (1990)

2. An, J., Chen, Y.: Region based image segmentation using a modified Mumford-Shah algorithm. In: Proc. Scale Space Varional Methods in Computer Vision, pp. 733-742 (2007)

3. Baldo, S.: Minimal interface criterion for phase transitions in mixtures of Cahn-Hilliard fluids. Annals of Institute Henri Poincare 7, 67-90 (1990)

4. Chan, T., Vese, L.: Active contours without edges. IEEE Transaction on Image Processing 10, 266-277 (2001)

5. Esedoglu, S., Tsai, R.: Threshold dynamics for the piecewise constant Mumford-Shah fuctional. Computational and Applied Mathematics Report. 04-63 UCLA (2004)

6. Li, C., Kao, C., Gore, J., Ding, Z.: Implicit active contours driven by local binary fitting energy. In: Proc. IEEE Computer Society Conference on Computer Vision and Pattern Recognition, IEEE Computer Society Press, Los Alamitos (2007)

7. Lie, J., Lysaker, M., Tai, X.: A binary level set model and some aplications to Mumford-Shah segmentation. Computational and Applied Mathematics Report. vol. 31 (2004)

8. Modica, L.: The gradient theory of phase transitions and the minimal interface criterion. Archive for Rational Mechanics and Analysis 98, 123-142 (1987)

9. Mumford, D., Shah, J.: Optimal approximations by piecewise smooth functions and associated variational problems. Communications on Pure and Applied Mathematics 42, 577-685 (1989)

10. Osher, S., Fedkiw, R.: Level set methods and dynamic implicit surfaces. Springer, New York (2003)

11. Piovano, J., Rousson, M., Papadopoulo, T.: Efficient segmentation of piecewise smooth images. In: Proc. Scale Space Varional Methods in Computer Vision, pp. 709-720 (2007)

12. Shen, J.: $\Gamma$-Convergence approximation to piecewise constant Mumford-Shah segmentation. In: Blanc-Talon, J., Philips, W., Popescu, D.C., Scheunders, P. (eds.) ACIVS 2005. LNCS, vol. 3708, pp. 499-506. Springer, Heidelberg (2005)

13. Wang, M., Zhou, S.: Phase field: A variational method for structural topology optimization. Computer Modeling in Engineering \& Science 6, 547-566 (2004)

14. Hou, Z.: A review on MR image intensity inhomogeneity correction. International Journal of Biomedical Imaging, 1-11 (2006)

15. Zhang, Y., Brady, M., Smith, S.: Segmentation of brain MR images through a hidden Markov random field model and the expectation-maximization algorithm. IEEE Transactions on Medical Imaging 20, 45-57 (2001) 